\title{
CHARACTERIZATION OF LIPSCHITZ SPACES ON COMPACT LIE GROUPS
}

\author{
DASHAN FAN and ZENGFU XU
}

(Received 13 April 1992;revised 7 august 1992)

Communicated by I. Raeburn

\begin{abstract}
Lipschitz spaces are important function spaces with relations to $H^{p}$ spaces and Campanato spaces, the other two important function spaces in harmonic analysis. In this paper we give some characterizations for Lipschitz spaces on compact Lie groups, which are analogues of results in Euclidean spaces.
\end{abstract}

1991 Mathematics subject classification (Amer. Math. Soc.): 43A15, 22E30, 41 A25, 41A30.

\section{Introduction and Notation}

Lipschitz spaces are very important function spaces with close relations to $H^{p}$ spaces and Campanato spaces, the other two important function spaces in harmonic analysis. The theory of these spaces on Euclidean spaces has been fruitfully developed by several authors (see [2, 3, 4]). In this paper we consider Lipschitz spaces on compact Lie groups and give some characterizations for these spaces, which are analogues of the results in the case of Euclidean spaces.

Let $G$ be a compact connected semisimple Lie group of dimension $n$. Choose $T \subset G$ to be a fixed maximal torus of $G$ with $\operatorname{dim} T=\operatorname{rank} G=l$. Let $\mathbf{g}$ and $\mathbf{t}$ denote the Lie algebras of $G$ and $T$ respectively, and $\mathbf{g}_{c}$ and $\mathbf{t}_{c}$ their complexification. If $\Delta$ is the set of roots of $\left(\mathbf{g}_{c}, \mathbf{t}_{c}\right)$ we choose in $\Delta$ a system $\Delta^{+}$of positive roots. Write $m=$ card $\Delta^{+}$and $n=2 m+l$. We choose inner product $(\cdot, \cdot)$ on $\mathbf{g}$ which is invariant under the adjoint action of $G$ on g. Put $(\cdot, \cdot)^{1 / 2}=|\cdot|$, so that $|\cdot|$ is a norm on g. Let $d$ be the geodesic metric on $G$ associated with $(\cdot, \cdot)$, so that for small $t$, $d(\exp t X, e)=|t||X|$, for $X \in \mathbf{g}$, where $e$ is the identity of $G$. Let $\hat{G}$ be the set of equivalence classes of irreducible unitary representations of $G$ and denote by $\chi_{\lambda}$ and $d_{\lambda}$ the character and dimension of the unitary representation $U_{\lambda}$ corresponding to $\lambda \in \hat{G}$.

(C) 1995 Australian Mathematical Society 0263-6115/95 \$A2.00+0.00 
Fix once and for all an orthonormal basis $\left\{X_{1}, \cdots, X_{n}\right\}$ of g. Set $X^{\gamma}=X_{1}{ }^{\gamma_{1}} \cdots X_{n}{ }^{\gamma_{n}}$ for each positive integral $n$-tuple $\gamma=\left(\gamma_{1}, \cdots, \gamma_{n}\right)$.

In the sequel we denote by $c$ a positive constant which may change from line to line.

\section{Definitions and main results}

Suppose $\alpha \geq 0$. We introduce Lipschitz spaces on $G$.

DEFINITION 1. Denote by $\dot{\Lambda}_{\alpha}$ the homogeneous Lipschitz spaces and $\Lambda_{\alpha}$ the (inhomogeneous) Lipschitz spaces.

(i) For $0<\alpha<1, \dot{\Lambda}_{\alpha}=\left\{f:|f(x)-f(y)| \leq c d(x, y)^{\alpha}, x, y \in G\right\}$ and $\|f\|_{\dot{\Lambda}_{\alpha}}$ is the infimum of all $c$ for which the above estimate holds.

(ii) For $\alpha=1, \dot{\Lambda}_{1}=\left\{f:\|f\|_{\dot{\Lambda}_{1}}<\infty\right\}$, where

$$
\|f\|_{\Lambda_{1}}=\sup \left\{\frac{\left|f(x y)-2 f(x)+f\left(x y^{-1}\right)\right|}{d(y, e)}: x, y \in G, y \neq e\right\} .
$$

(iii) For $\alpha>1$, let $k$ be the positive integer such that $k<\alpha \leq k+1$. Then $\dot{\Lambda}_{\alpha}=\left\{f:\|f\|_{\dot{\Lambda}_{\alpha}}<\infty\right\}$ where

$$
\|f\|_{\dot{\Lambda}_{\alpha}}=\sum_{|\gamma|=k}\left\|X^{\gamma} f\right\|_{\dot{\Lambda}_{\alpha-k}} .
$$

(iv) For any $\alpha>0$ we define $\|f\|_{\Lambda_{\alpha}}=\|f\|_{1}+\|f\|_{\Lambda_{\alpha}}$ and $\Lambda_{\alpha}=\left\{f \in L^{1}(G)\right.$ : $\left.\|f\|_{\Lambda_{\alpha}}<\infty\right\}$.

For an integer $k \geq 0$ we define $A_{k}$ and $\tilde{A}_{k}$ by

$$
\begin{aligned}
& A_{k}=\left\{\varphi \in S(\mathbf{t}): \begin{array}{l}
\varphi \text { radial, } \operatorname{supp} \varphi \subset\{H:|H| \leq 1\} \\
\text { and } \int_{t} \varphi(H) H^{\gamma} d H=0 \text { for all }|\gamma| \leq k
\end{array}\right\}, \\
& \tilde{A}_{k}=\left\{\varphi \in A_{k}: \int_{0}^{\infty} \hat{\varphi}(t H)^{2} t^{-1} d t=1, H \neq 0\right\}
\end{aligned}
$$

where $|\gamma|=\gamma_{1}+\cdots+\gamma_{n}$ for a positive integral $n$-tuple $\gamma=\left(\gamma_{1}, \ldots, \gamma_{n}\right)$. By [3] we know $\tilde{A}_{k}$, and therefore $A_{k}$, are not empty.

For $t>0$ let $\varphi_{t}(x)=\sum_{\lambda \in \hat{G}} \hat{\varphi}(t|\lambda+\beta|) d_{\lambda} \chi_{\lambda}(x)$, where $\beta=\sum_{\alpha \in \Delta^{+}} \alpha / 2$.

DEFINITION 2. For $0<\alpha<1$ and $\varphi \in \tilde{A}_{o}$ we set $\|f\|_{\dot{B}_{\alpha}}=\sup \left\{\left(t^{-\alpha}\left|\varphi_{t} * f(x)\right|\right.\right.$ : $x \in G, t>0\},\|f\|_{B_{\alpha}}=\|f\|_{1}+\|f\|_{\dot{B}_{\alpha}}$, and define $\dot{B}_{\alpha}=\left\{f:\|f\|_{\dot{B}_{\alpha}}<\infty\right\}$ and $B_{\alpha}=\left\{f:\|f\|_{B_{\alpha}}<\infty\right\}$. 
A standard argument gives

THEOREM 1. For $0<\alpha<1$ we have $\dot{\Lambda}_{\alpha}=\dot{B}_{\alpha}$ and $\Lambda_{\alpha}=B_{\alpha}$.

The following theorem gives a characterization of $\Lambda_{\alpha}$, for all $\alpha>0$.

THEOREM 2. Let $\alpha>0$, and $k \in \mathbb{Z}, k \geq[\alpha]$ where $[\alpha]$ denotes the greatest integer part of $\alpha$. Then the following are equivalent.

(i) $f \in \Lambda_{\alpha}$.

(ii) For all $\varphi \in A_{2 k+n}, \sup \left\{t^{-\alpha}\left|\varphi_{t} * f(x)\right|: x \in G, t>0\right\} \leq c_{\varphi, f}$.

(iii) There exists $\varphi \in \tilde{A}_{2 k+n}$ such that $\sup \left\{t^{-\alpha}\left|\varphi_{t} * f(x)\right|: x \in G, t>0\right\} \leq c_{\varphi, f}$. Moreover, if we define $\|f\|_{B_{\alpha}}=\|f\|_{1}+\sup _{(x, t)} t^{-\alpha}\left|\varphi_{t} * f(x)\right|$ for some $\varphi \in A_{2 k+n}$ then $\|f\|_{\Lambda_{\alpha}} \sim\|f\|_{B_{\alpha}}$.

Let exp be the exponential map of $g$ to $G$. Then exp is an analytic diffeomorphism on an open neighbourhood of the origin of g. Choose $\epsilon_{0}$ and $\epsilon_{0}{ }^{\prime}$ to be the maximal positive numbers so that exp is such that a diffeomorphism of $B\left(0, \epsilon_{0}^{\prime}\right)$ onto $B\left(0, \epsilon_{0}\right)$. For a positive integer $k$ we set

$$
P_{k}=\left\{\begin{array}{ll}
p: \begin{array}{l}
p(x)=q\left(\exp ^{-1} x\right) \text { for } x \in B\left(e, \epsilon_{0}\right), \\
\mathrm{q} \text { is a polynomial on } \mathrm{g} \text { with degree } \leq k
\end{array}
\end{array}\right\} .
$$

DEFINITION 3. Let $1 \leq q<\infty, \lambda>0$ and $k \geq 0, k \in \mathbb{Z}$. The Campanato spaces are defined as $L_{k}{ }^{q, \lambda}(G)=\left\{f:\|f\|_{L_{k}}^{q, \lambda}<\infty\right\}$ where

$$
\|f\|_{L_{k}^{q, \lambda}}=\|f\|_{1}+\sup _{x \in G, 0<r<\epsilon_{0}} \inf _{p \in P_{k}}\left\{|B(x, r)|^{-\lambda / n} \int_{B(x, r)}\left|f(y)-p\left(x^{-1} y\right)\right|^{q} d y\right\}
$$

and $|B(x, r)|$ is the Haar measure of the ball $B(x, r)=\{y: d(y, x)<r\}$.

We now state a theorem which gives the relationship between the Lipschitz spaces, the Hardy spaces $H^{p}(G)$ and the Campanato spaces.

THeOREM 3. Let $1 \leq q<\infty, \alpha>0, k=[\alpha]$ and $p=n /(n+\alpha)$. Then the following are equivalent.

(a) $f \in \Lambda_{\alpha}$.

(b) There exists $c>0$ such that for each $x \in G$ and $0<r<\epsilon_{0}$, there is a $p_{k}=p_{x, r, k} \in P_{k}$ satisfying

$$
\sup _{y \in B(x, r)}\left|f(y)-p_{k}\left(x^{-1} y\right)\right| \leq c r^{\alpha} .
$$

(c) $f \in L_{k}^{q, n+\alpha q}(G)$.

(d) $f \in\left(H^{p}\right)^{*}$, where $\left(H^{p}\right)^{*}$ denotes the dual of $H^{p}(G)$. 


\section{Proof of theorems}

To prove Theorem 2 we first establish a lemma.

LEMMA. Suppose $\alpha, k$ are as in Theorem 2. Then the following statements are equivalent:

(a) For all $\varphi \in A_{2 k}$, $\sup _{x, t} t^{-\alpha}\left|\varphi_{t} * f(x)\right| \leq c_{\varphi, f}$.

(b) There exists some $\varphi \in \tilde{A}_{2 k}$ such that $\sup _{x, t} t^{-\alpha}\left|\varphi_{t} * f(x)\right| \leq c_{\varphi, f}$.

Proof. We need only prove (b) implies (a). Suppose that $\varphi \in \tilde{A}_{2 k}$ such that $\sup _{x, t} t^{-\alpha}\left|\varphi_{t} * f(x)\right| \leq c_{\varphi, f}$. Then $f(x)=\int_{0}^{\infty} \varphi_{t} * \varphi_{t} * f(x) t^{-1} d t$. For any $\psi \in A_{2 k}$ we have

$$
\begin{aligned}
\psi_{t} * f(x) & =\int_{0}^{\infty} \psi_{t} * \varphi_{s} * \varphi_{s} * f(x) s^{-1} d s \\
& =\int_{0}^{t} \psi_{t} * \varphi_{s} * \varphi_{s} * f(x) s^{-1} d s+\int_{t}^{\infty} \psi_{t} * \varphi_{s} * \varphi_{s} * f(x) s^{-1} d s \\
& =I_{1}(x, t)+I_{2}(x, t)
\end{aligned}
$$

It is easy to check that for any $\varphi \in A_{k}, \varphi_{t} \in L^{1}(G), \operatorname{supp} \varphi_{t} \subset B(e, t)$, the Poisson formula gives

$$
\varphi_{t}(x)=\varphi_{t}(\exp H)=c t^{-1} D(\exp H)^{-1} \prod_{\alpha \in \Delta+} \frac{\partial}{\partial \alpha}\left(\varphi\left(\frac{H}{t}\right)\right),
$$

where $\exp H \in T$ is the only element conjugate to $x$ and $D(\exp H)$ is the Weyl function

$$
D(x)=D(\exp H)=(-2 i)^{m} \prod_{\alpha \in \Delta+} \sin \left(\frac{i \alpha(H)}{2}\right) .
$$

So we can easily deduce that for all $x \in G, t>0,\left|I_{1}(x, t)\right| \leq c_{\varphi, \psi} t^{\alpha}$.

For $I_{2}$, we can assume $t<\epsilon_{0}$ since $\left|I_{2}(x, t)\right| \leq c_{\varphi, \psi, f} \leq c_{\varphi, \psi, f} t^{\alpha}$ otherwise. Denote by $\alpha_{1}, \ldots, \alpha_{m}$ all the positive roots. Then

$$
D(x)=D(\exp H)=G_{k}(H)+F_{k}(H),
$$

where

$$
G_{k}(H)=c_{m} \sum_{j=0}^{\left\lfloor\frac{k}{2}\right]}(-1)^{j} 2^{m+2 j} \sum_{J \in A_{j}} \prod_{i=1}^{m} \frac{1}{j_{i} !} \alpha_{i}(H)^{j_{i}},
$$

with $J=\left(j_{1}, \ldots, j_{m}\right)$ and

$$
A_{j}=\left\{\left(j_{1}, \ldots, j_{m}\right): j_{1}+\cdots+j_{m}=2 j+m, j_{1}, \ldots, j_{m} \text { odd }\right\},
$$


and where $F_{k}$ has the following estimate:

$$
F_{k}(H)=O\left(|H|^{m+2\left[\frac{k}{2}\right]+2}\right),|H|<\epsilon_{0} .
$$

Write

$$
\begin{aligned}
I_{2}(x, t) & =\int_{t}^{\infty} \Phi_{k, t}^{(1)} * \varphi_{s} * \varphi_{s} * f(x) s^{-1} d s+\int_{t}^{\infty} \Phi_{k, t}^{(2)} * \varphi_{s} * \varphi_{s} * f(x) s^{-1} d s \\
& =J_{1}(x, t)+J_{2}(x, t)
\end{aligned}
$$

where

$$
\begin{aligned}
& \Phi_{k, t}^{(1)}(x)=\Phi_{k, t}^{(1)}(\exp H)=\Psi_{t}(\exp H) G_{k}(H) D(\exp H)^{-1} \\
& \Phi_{k, t}^{(2)}(x)=\Phi_{k, t}^{(2)}(\exp H)=\Psi_{t}(\exp H) F_{k}(H) D(\exp H)^{-1}
\end{aligned}
$$

Let $h_{s}(x)=\varphi_{s} * \varphi_{s} * f(x)$. Observe that there exists $q \in \mathbb{Z}^{+}$such that $\|\hat{f}(\lambda)\| \leq$ $c_{f}|\lambda+B|^{q}$ and $\hat{\varphi} \in S(t)$. We then have

$$
\left\|h_{s}\right\|_{\infty} \leq\left\{\begin{array}{lll}
c_{\varphi, f} s^{\alpha} & \text { if } & s<\epsilon_{0} \\
c_{\varphi, f} s^{-n-q} & \text { if } & s>\epsilon_{0}
\end{array}\right.
$$

From this and (1), (3) we have $\left|J_{2}(x, t)\right| \leq c_{\varphi, \psi, f} t^{\alpha}$.

Let $Y_{1}, Y_{2}, \ldots, Y_{1}$ be an orthonormal basis of $\mathrm{t}$. Then for $y \in B\left(e, \epsilon_{0}\right)$ there exist $\tilde{y} \in G$ and $H \in \mathbf{t},|H|<\epsilon_{0}$ such that

$$
y^{-1}=\exp (\operatorname{Ad}(\tilde{y}) \mathrm{H})=\exp \left(\sum_{j=1}^{l} H_{j} \operatorname{Ad}(\tilde{y}) Y_{j}\right) .
$$

Thus, by the Taylor series expansion (see [5])

$$
h_{s}\left(x y^{-1}\right)=\sum_{\gamma} \frac{\tilde{X}(\gamma) h_{s}(x)}{\gamma_{1} ! \ldots \gamma_{l} !} H_{1}^{\gamma_{1}} H_{2}^{\gamma_{2}} \cdots H_{l}^{\gamma_{1}}, y \in B\left(e, \epsilon_{0}\right), x \in G,
$$

where $\tilde{X}(\gamma)$ is the coefficient of $H_{1}{ }^{\gamma_{1}} H_{2}{ }^{\gamma_{2}} \ldots H_{l}{ }^{\gamma_{1}}$ in the formal polynomial

$$
\frac{\gamma_{1} ! \ldots \gamma_{l} !}{|\gamma| !}\left(H_{1} \operatorname{Ad}(\tilde{y}) Y_{1}+\cdots+H_{l} \operatorname{Ad}(\tilde{y}) Y_{l}\right)^{|\gamma|}
$$

Observe that $\operatorname{Ad}(\tilde{y}) Y_{j} f(x)=\sum_{i=1}^{n} c_{i j}(\tilde{y}) X_{i} f(x)$, where the $c_{i j}(\tilde{y})$ satisfy

$$
\sum_{i=1}^{n}\left|c_{i j}(\tilde{y})\right|^{2}=1 \text {, }
$$


and

$$
\left|X^{\gamma} h_{s}(w)\right| \leq \begin{cases}c_{\varphi, f} s^{\alpha-|\gamma|} & \text { if } \quad s<\epsilon_{0} \\ c_{\varphi, f} s^{-(q+n)} & \text { for some } q \in \mathbb{Z}^{+} \text {otherwise. }\end{cases}
$$

By (1) and (4) we then can deduce that

$$
\left|J_{1}(x, t)\right| \leq \int_{t}^{\infty} \int_{G}\left|\Phi_{k, t}^{(1)}(y)\right| \sup _{|\gamma| \leq k+1, w \in B(x, t)}\left|X^{\gamma} h_{s}(w)\right| d y \leq c_{\varphi, \psi, f} t^{\alpha}
$$

which completes the proof of the Lemma.

PROOF OF THEOREM 2. In view of Theorem 1 and the lemma we are reduced to proving the equivalence of (i) and (ii) for $\alpha \geq 1$. We begin with the implication (i) implies (ii) for $\alpha=1$. Let $f \in \Lambda_{1}$. For any $\varphi \in A_{2 k+n}$ it is easy to check that $\left|\varphi_{t} * f(x)\right| \leq c\|f\|_{\Lambda_{1}} t$ for $t>\epsilon_{0}$, and $\varphi_{t} * f(x)=\int_{G} \varphi(y) f(x y) d y$. By (2) and (4) we have, for $t<\epsilon_{0}$,

$$
\left|\varphi_{t} * f(x)\right| \leq c\|f\|_{\Lambda_{1}} t+\int_{G}\left(\left|f\left(x y^{-1}\right)\right|+|f(x y)|\right)\left|\Phi_{2 k+n, t}^{(2)}(y)\right| d y
$$

then we use (1) and (3) to obtain the desired estimate.

Now we prove (ii) implies (i) for $\alpha=1$. We can assume $\|f\|_{B_{1}}<\infty$ for $\varphi \in \tilde{A}_{2 k+n}$. Then for any $y \in G$ the Calderon formula gives

$$
\Delta_{y}{ }^{2} f(x)=\int_{0}^{d(e, y)} \Delta_{y}{ }^{2} \varphi_{s} * \varphi_{s} * f(x) s^{-1} d s+\int_{d(e, y)}^{\infty} \Delta_{y}{ }^{2} \varphi_{s} * \varphi_{s} * f(x) s^{-1} d s
$$

where

$$
\Delta_{y}^{2} f(x)=f(x y)-2 f(x)+f\left(x y^{-1}\right) .
$$

Observe that $\left\|X^{\gamma} \varphi_{t}\right\|_{1} \leq c_{\varphi} t^{-|\gamma|}$ for $t<\epsilon_{0}$, and that $\left\|X^{\gamma} \varphi_{t}\right\|_{1}=O(1)$ otherwise. We then have

$$
\begin{aligned}
\left|\Delta_{y}{ }^{2} f(x)\right| & \leq c_{\varphi}\|f\|_{B_{1}} d(y, e)+\int_{d(y, e)}^{\infty} d(y, e) \sum_{|\gamma|=2}\left\|X^{\gamma} \varphi_{s} * \varphi_{s} * f\right\|_{\infty} s^{-1} d s \\
& \leq c_{\varphi}\|f\|_{B_{1}} d(y, e)
\end{aligned}
$$

which shows $\|f\|_{\Lambda_{1}} \leq c_{\varphi}\|f\|_{B_{1}}$. Thus the theorem follows for $\alpha=1$.

For $\alpha>1$ choose $j \in \mathbb{Z}, 0 \leq j \leq k$ such that $k-\alpha<j \leq k-\alpha+1$ and $\alpha-1 \leq|\gamma|=k-j<\alpha$. A similar argument as for the case $\alpha=1$ gives $X^{\gamma} f \in \dot{\Lambda}_{\alpha-|\gamma|}$ if $f \in B_{\alpha}$ and $\|f\|_{\Lambda_{\alpha}} \leq c\|f\|_{B_{\alpha}}$. All that remains now is (i) implies 
(ii). Suppose $f \in \Lambda_{\alpha}, \alpha>1$. Then $X^{\gamma} f \in \dot{B}_{\alpha-|\gamma|}$ by Theorem 1. Choose $\varphi \in \tilde{A}_{2}$ to obtain

$$
\begin{aligned}
\varphi_{t} * f(x)= & \int_{0}^{t} \Phi_{k+n, t}^{(1)} \varphi_{s} * \varphi_{t} * f(x) s^{-1} d s+\int_{t}^{\infty} \Phi_{k+n, t}^{(1)} \varphi_{s} * \varphi_{s} * f(x) s^{-1} d s \\
& +\int_{0}^{t} \Phi_{k+n, t}^{(2)} \varphi_{s} * \varphi_{s} * f(x) s^{-1} d s+\int_{t}^{\infty} \Phi_{k+n, t}^{(2)} \varphi_{s} * \varphi_{t} * f(x) s^{-1} d s \\
= & \sum_{i=1}^{4} \sigma_{i}(x, t)
\end{aligned}
$$

where $\Phi_{k+n, t}^{(1)}, \Phi_{k+n, t}^{(2)}$ are as in (4). We may, and do, assume that $t<\epsilon_{0}$. Observe that $\left\|\varphi_{s}\right\|_{\infty} \leq c_{\varphi} s^{-n}$. Thus $\left|\sigma_{i}(x, t)\right| \leq c_{\varphi}\|f\|_{\Lambda_{\alpha}} t^{\alpha}$ for $i=3,4$. Let $h_{1}(x)=\varphi_{s} * \varphi_{s} * f(x)$ and $h_{2}(x)=\varphi_{s} * \varphi_{t} * f(x)$. Then by (1), (2) and (4)

$\left|\sigma_{2}(x, t)\right| \leq c t^{k+1} \int_{t}^{\infty} \int_{B(e, t)}\left|\Phi_{k+n, t}^{(1)}(y)\right| \sup _{|v|=k+1, w \in B(x, t)}\left|X^{\nu} h_{1}(w)\right| d y s^{-1} d s \leq c\|f\|_{\Lambda_{\alpha}} t^{\alpha}$

and

$$
\left|\sigma_{1}(x, t)\right| \leq c_{\varphi} \int_{0}^{t} s^{|\gamma|} \sup _{|\gamma|=k-j, w \in B(x, r)}\left|X^{\gamma} h_{2}(w)\right| s^{-1} d s \leq c\|f\|_{\Lambda_{\alpha}} t^{\alpha} .
$$

This proves (i) implies (ii) for $\alpha>1$ and completes the proof of Theorem 2.

ProOF OF THEOREM 3. The equivalence of (c) and (d) is proved in [1]. Now we prove (b) implies (c) and (d) implies (a) implies (b).

Suppose that (b) is true. Then $f \in L^{\infty}(G)$ due to the compactness of G. Let $p_{k}$ be the polynomial as given in (b) for a fixed $x \in G$ and $0<r<\epsilon_{0}$. We then have

$$
|B(x, r)|^{-(n+\alpha q) / n} \int_{B(x, r)}\left|f(y)-p_{k}\left(x^{-1} y\right)\right|^{q} d y \leq c
$$

which implies (c).

To prove (d) implies (a) we need to show that, for some $\varphi \in \tilde{A}_{4 k+n},\left\|\varphi_{t} * f\right\|_{\infty} \leq c t^{\alpha}$ if $f \in\left(H^{P}\right)^{*}$. Observe that $\left\|\varphi_{t}\right\|_{H^{p}} \leq c \sum_{\lambda \in \hat{G}}|\hat{\phi}(t|\lambda+\beta|)|^{2} d_{\lambda}{ }^{2}$. Hence, for $t \geq \epsilon_{0}$, $\left\|\varphi_{t} * f\right\|_{\infty} \leq c\|f\|_{\left(H^{P}\right)^{*}} t^{\alpha}$.

We now assume $t<\epsilon_{0}$ and write

$$
\varphi_{t} * f(x)=\Phi_{2 k+n, t}^{(1)} * f(x)+\Phi_{2 k+n, t}^{(2)} * f(x)
$$

where $\Phi_{2 k+n, t}^{(1)}, \Phi_{2 k+n, t}^{(2)}$ are as in (4). By (3), $\left\|\Phi_{2 k+n, t}^{(2)} * f\right\|_{\infty} \leq c\|f\|_{\left(H^{\rho}\right)^{*}} t^{\alpha}$. Observe that for any $p_{2 k+1} \in \mathbf{P}_{2 k+1}$, 


$$
\begin{aligned}
\int_{G} t^{-\alpha} \Phi_{2 k+n, t}^{(1)}(x) p_{2 k+1}(x) d x & \\
= & c t^{-\alpha-l} \int_{G / T}\left\{\int_{B(0, t)} G_{2 k+n}(H)_{q 2 k+1}\left(\sum_{j=1}^{n}\left(\sum_{i=1}^{n} c_{i j}(y) H_{i}\right) X_{j}\right) \times\right. \\
& \left.\times \prod_{\alpha \in \Delta^{+}} \frac{\partial}{\partial \alpha}\left(\varphi\left(\frac{H}{t}\right)\right) d H\right\} d y=0 .
\end{aligned}
$$

Moreover supp $\left\{t^{-\alpha} \Phi_{2 k+n, t}^{(1)}\right\} \subset B(e, t)$, and $\left\|t^{-\alpha} \Phi_{2 k+n, t}^{(1)}\right\|_{\infty} \leq c t^{-n / p}$. Thus $t^{-\alpha} \Phi_{2 k+n, t}^{(1)}$ is a $(p, \infty)$ atom which implies $\left\|\Phi_{2 k+n, t}^{(1)} * f\right\|_{\infty} \leq c\|f\|_{\left(H^{p}\right)}^{*} t^{\alpha}$. The implication (d) implies (a) is therefore proved.

It remains to prove (a) implies (b). Suppose first that $\alpha$ is not an integer. Then $k<\alpha<k+1$. For any $x \in G$ and $0<r<\epsilon_{0}$, if $y \in B(x, r)$ then there exists a $z_{y} \in B(x, r)$ such that

$$
f(y)=\sum_{|\gamma| \leq k-1} X(\gamma) f(x) P_{\gamma}\left(x^{-1} y\right)+\sum_{|\gamma|=k} X(\gamma) f\left(z_{y}\right) P_{\gamma}\left(x^{-1} y\right)
$$

where $P_{\gamma} \in P_{|\gamma|}$. Let $p_{k}\left(x^{-1} y\right)=\sum_{|\gamma| \leq k} X(\gamma) f(x) P_{\gamma}\left(x^{-1} y\right)$ for $y \in B(x, r)$. Then

$$
\left|f(y)-p_{k}\left(x^{-1} y\right)\right| \leq c \sum_{|\gamma|=k}\left|X(\gamma) f(x)-X(\gamma) f\left(z_{y}\right)\right|\left|P_{\gamma}\left(x^{-1} y\right)\right| \leq c\|f\|_{\Lambda_{\alpha}} r^{\alpha}
$$

which gives $\|f\|_{L_{k}{ }^{q, \lambda}} \leq c\|f\|_{\Lambda_{\alpha}}$ for $k<\alpha<k+1$. Now we consider the case when $\alpha$ is an integer. Firstly we suppose $\alpha=1$. Taking a radial function $\eta \in$ $C^{\infty}(\mathbf{t})$ satisfying supp $\eta \subset B\left(O, \frac{1}{2}\right)$ and $\int_{t} \eta(H) d H=1$ we then define $\eta_{r}(x)=$ $\hat{\eta}(r|\beta|) \sum_{\lambda \in \hat{G}} \hat{\eta}(r|\lambda+\beta|) d_{\lambda} \chi_{\lambda}(x)$ and $N_{r}=\eta_{r} * \eta_{r}$. It is easy to verify that $N_{r}$ has the following properties.
(i) $\int_{G} N_{r}(x) d x=1$,
(ii) $N_{r}\left(x^{-1}\right)=N_{r}(x)$,
(iii) $N_{r}$ is central, and
(iv) $\int_{G} X^{\gamma} N_{r}(x) d x=0$ for $|\gamma|=2$.

Thus

$$
\begin{aligned}
\left|f(y)-N_{r} * f(y)\right| & =\frac{1}{2}\left|\int_{G}\left[2 f(y)-f\left(y z^{-1}\right)-f(y z)\right] N_{r}(z) d z\right| \\
& \leq \frac{1}{2}\|f\|_{\Lambda_{1}} \int_{G} \int_{G}\left[d\left(z w^{-1}, e\right)+d(w, e)\right]\left|\eta_{r}(w) \eta_{r}\left(w^{-1} z\right)\right| d w d z \\
& \leq c\|f\|_{\Lambda_{1}} r .
\end{aligned}
$$

Given $x \in G$ and $0<r<\epsilon_{0}$, for any $y \in B(x, r)$ we write

$$
N_{r} * f(y)=\sum_{|\gamma| \leq 1} X(\gamma)\left(N_{r} * f\right)(x) P_{\gamma}\left(x^{-1} y\right)+\sum_{|\gamma|=2} X(\gamma)\left(N_{r} * f\right)\left(z_{y}\right) P_{\gamma}\left(x^{-1} y\right)
$$


where $z_{y} \in B(x, r)$ as before. Thus we are reduced to proving that, for $f \in \Lambda_{1}$,

$$
\sup _{w \in B(x, r), 1 \leq i, j \leq n}\left|X_{i} X_{j}\left(N_{r} * f\right)(w)\right| \leq c r^{-1}\|f\|_{\Lambda_{1}},
$$

where $c$ is a constant independent of $x$ and $r$. To see this let $\mathbf{D}(G)$ be the algebra of all left-invariant differential operators on $G$. We recall that $g$ can be regarded as a Lie subalgebra of $\mathbf{D}(G)$ with $[X, Y]=X Y-Y X$, where $[X, Y]$ is the bracket of $X$ and $Y$. Then we have

$$
X_{i} X_{j} N_{r}\left(y^{-1}\right)=X_{i} X_{j} N_{r}(y)+\sum_{k=1}^{n} c_{k}^{i j} X_{k} N_{r}(y)
$$

and

$$
\begin{aligned}
X_{i} X_{j} N_{r} * f(y)= & \frac{1}{2} \int_{G} X_{i} X_{j} N_{r}(y)\left(f(x y)+f\left(x y^{-1}\right)-2 f(x)\right) \\
& -\sum_{s=1}^{n} c_{s}{ }^{i j} X_{s} N_{r} * f(y) .
\end{aligned}
$$

Observe that $\Lambda_{1} \subset \Lambda_{1 / 2}$ and we have

$$
\left|X_{s} N_{r} * f(y)\right| \leq\|f\|_{\Lambda_{1}} \int_{G}\left|X_{s} N_{r}\left(y z^{-1}\right)\right| d(y, z)^{1 / 2} d z \leq c\|f\|_{\Lambda_{1}} r^{-1} .
$$

Thus (6) follows from (8).

Now we consider the case when $\alpha=k \in \mathbb{Z}^{+}$and $k \geq 2$. Suppose $f \in \Lambda_{k}$. Then $f \in L^{1}$ and

$$
\left\|X^{\gamma}\left(f-N_{r} * f\right)\right\|_{\infty} \leq c\left\|X^{\gamma} f\right\|_{\dot{\Lambda}_{1}} r=c\|f\|_{\Lambda_{\alpha}} r
$$

for all $\gamma,|\gamma|=k-1$. On the other hand, Taylor's formula gives

$$
f(y)-N_{r} * f(y)=q_{k-2}\left(x^{-1} y\right)+\sum_{|\gamma|=k-1} X(\gamma)\left(f-N_{r} * f\right)\left(z_{y}\right) P_{\gamma}\left(x^{-1} y\right)
$$

for each $y \in B(x, r)$ and some $z_{y} \in B(x, r)$, where $q_{k-2} \in \mathbf{P}_{k-2}$ and $P_{r} \in \mathbf{P}_{|y|}$. By (8),

$$
\left|f(y)-N_{r} * f(y)-q_{k-2}\left(x^{-1} y\right)\right| \leq c\|f\|_{\Lambda_{\alpha}} r^{\alpha} .
$$

For each $y \in B(x, r)$, there is also a $z^{\prime}{ }_{y} \in B(x, r)$ such that

$$
N_{r} * f(y)=\tilde{q}_{k}\left(x^{-1} y\right)+\sum_{|\gamma|=k+1} X(\gamma)\left(N_{r} * f\right)\left(z^{\prime}{ }_{y}\right) P_{\gamma}\left(x^{-1} y\right)
$$


where

$$
\tilde{q}_{k}\left(x^{-1} y\right)=\sum_{|\gamma| \leq k} X(\gamma)\left(N_{r} * f\right)(x) P_{\gamma}\left(x^{-1} y\right) .
$$

Write $|\gamma|=\left|\gamma_{1}\right|+\left|\gamma_{2}\right|$ where $\left|\gamma_{1}\right|=2$. Then

$$
\left|N_{r} * f(y)-\tilde{q}_{k}\left(x^{-1} y\right)\right| \leq c \sum_{\left|\gamma_{1}\right|=2,\left|\gamma_{2}\right|=k-1}\left\|X^{\gamma_{1}} N_{r} * X^{\gamma_{2}} f\right\|_{\infty} r^{\alpha+1} \leq c\|f\|_{\Lambda_{\alpha}} r^{\alpha}
$$

which together with (9) gives the desired result. The theorem is therefore proved.

\section{References}

[1] D. Fan, Hardy spaces on compact Lie groups (PhD thesis, Washington University, St. Louis, 1990).

[2] S. G. Krantz, 'Structure and interpolation theorems for certain Lipschitz spaces and estimates for the $\bar{\partial}$ equation', Duke Math. J. 43 (1976), 417-439.

[3] M. Frazier, B. Jawerth and G. Weiss, 'Littlewood-Paley theory and the study of function spaces', CBMS Regional Conf. Ser. in Math. 79 (Conf. Board Math. Sci, Washington D.C).

[4] E. M. Stein, Singular integrals and differentiability properties of functions (Princeton University Press, Princeton, 1970).

[5] V. S. Varadarajan, Lie groups, Lie algebras and their representations (Springer, Berlin, 1984).

Department of Mathematical Sciences

University of Wisconsin

PO Box 413

Milwaukee, WI 53201 USA
Mathematics - MPS

Murdoch University

Murdoch, WA 6150

Australia 